\title{
Alla bisogna, abbiamo una risposta
}

\author{
Vittorio Coletti
}

PUBBLICATO: 13 OCTOBER 2020

\section{Quesito:}

Molti lettori ci chiedono se sia "corretto" usare la locuzione alla bisogna con il valore di "in caso di necessità, all'occorrenza' o se non sia piuttosto da ritenersi, come scrive uno di loro, "un'espressione datata, obsoleta", nonché incongrua visto che bisogno è maschile. Due domande riguardano la grafia: si scriva alla bisogna o all'abbisogna?

\section{Alla bisogna, abbiamo una risposta}

P er prima cosa: alla bisogna è una locuzione avverbiale, cioè un insieme di parole con funzione unitaria di avverbio; il suo significato è parafrasabile con "eventualmente, se occorre, all'occorrenza, in caso di necessità". Condivide questi significati col suo sinonimo che presenta il sostantivo al maschile, al bisogno, di cui è più rara e manierata, anche se neanche al bisogno è molto frequente, ancorché circolante pure nel linguaggio bancario.

E veniamo alle domande dei lettori, cominciando da quelle relative all'ortografia. Poiché le parole del sintagma sono il sostantivo femminile bisogna e la preposizione articolata alla, la grafia corretta è alla bisogna. Qualche pronuncia regionale e l'esistenza del verbo abbisognare possono aver favorito l'equivoco di quei lettori che si chiedevano se fosse da scrivere all'abbisogna invece che alla bisogna. Quanto alla parola, bisogna è, come il suo sinonimo bisogno, una voce di origine francese (cfr. il recente Roberta Cella, Francesismi, Milano, RCS MediaGroup, 2020): alle spalle di bisogno c'e besoing o besoign e a quelle dibisogna besoigne (oggi besogne), entrambi dal fràncone bisunnia (entrato nel latino medievale come bisonium), in cui si può ancora intravedere il francese soin 'cura, preoccupazione'. Non è il solo caso di due parole che, dallo stesso etimo o da due molto prossimi, sviluppano uguali significati l'una al maschile e l'altra al femminile (es.: tavolo-tavola, orecchio-orecchia, cioccolatocioccolata). Il sostantivo femminile bisogna è antico in italiano (anche nella forma con mancata chiusura della vocale protonica della sillaba iniziale: besogna) quanto il gemello maschile bisogno e lo usano, tra gli altri, Dante (Purgatorio XXXIII, 29: "Madonna, mia bisogna voi conoscete") e (ripetutamente) Boccaccio. Oggi la forma femminile è rara, ma lo era, rispetto a quella maschile, già anticamente, se è vero che un commentatore trecentesco di Dante, Francesco Buti, chiosa la citata "mia bisogna" del Poeta, con "li miei bisogni".

Il sostantivo femminile, che, secondo il Tommaseo, era giudicato antiquato già a fine Seicento, è classificato oggi dai dizionari come raro e letterario ed è di gran lunga scavalcato nell'uso dal suo gemello maschile. Anticamente la parola era usata anche al plurale, specie nel significato di 'faccende, affari, attività', con attenuazione del tratto della necessità, come ben spiega il Tommaseo (nel Tommaseo-Bellini alla voce) distinguendola da bisogno: "Bisogna è più affine a Faccenda che a Bisogno, appunto come il fr. distingue Besoin da Besogne; ma l'idea originaria è la ragione del modo, cioè di faccenda che debbasi fare, siccome porta anco il significato del gerundio Res facienda". Il valore deontico però è rimasto evidente negli usi più frequenti del sintagma alla bisogna, complemento col senso, anche esortativo, di 'al lavoro, all'opera da farsi' (D'Annunzio: "m'hai visto alla bisogna", "leste, alla bisogna") e con quello di 'a fare, a compiere il necessario' (Decamerone II, 9: "[Sicurano] pensando 
che alla bisogna non era da dare indugio... dal soldano impetrò che...", cioè, "pensando che a fare ciò che occorreva non bisognava indugiare, dal Sultano ottenne con le sue preghiere che...'), da cui si sono sviluppati il significato attuale e la funzione avverbiale odierna ('all'occorrenza'). Forse, questo valore e funzione si potrebbero già intravedere in un esempio trecentesco dalla Fiorita di Armanino registrato dal TLIO ("se difetto dal mio lato c'è stato, alla bisogna con questa mia spada lo scuserò", "se ho commesso un errore, se sarà necessario lo emenderò con la spada') e in uno dai Reggimenti e costumi di donna di Francesco da Barberino citato dal GDLI: "No gli dar latte di capra, se puoi, / e meno assai di cuccia...; / ver è che pure, alla bisogna, / quel della pecora più ti concedo", se non ci fosse il dubbio, specie nel secondo caso, che la punteggiatura (le virgole dell'inciso) dell'editore ottocentesco abbia eccessivamente attualizzato il valore del sintagma. Sono casi comunque che mostrano lo scivolamento del complemento verso l'avverbio sganciato dalla frase (da qui l'isolamento odierno della locuzione con virgole, nello scritto). Funzione e valore di complemento sono tuttavia rimasti, pur rari, sino ad oggi (o ieri), col senso già visto di 'a fare, a compiere il necessario', specie in associazione col verbo provvedere (provvedere alla bisogna, è ancora attestato da un paio di romanzi del Premio Strega tra gli anni Ottanta e Novanta, citati dal PTLLIN).

Oggi, come detto, la funzione della locuzione alla bisogna è quella di avverbio di frase, che comunica cioè una valutazione, la cautela del parlante su quanto egli stesso sta dicendo ed è perciò svincolato dalla frase in cui è inserito e ha il senso, qui dichiarato allinizio, di 'in caso di necessità', 'all'occorrenza', 'eventualmente'. Questo formato complesso e questo significato sono gli unici in cui ancora sopravvive il sostantivo femminile bisogna, che è ovviamente legittimo, anche se la parola (e, di conseguenza, la locuzione) è cosi rara da sembrare manierata ed è quindi da usare con parsimonia.

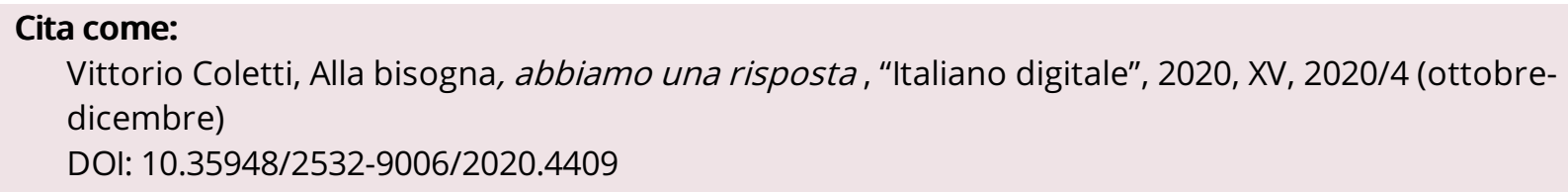

